\title{
The Use of $O$-(2-18 F-Fluoroethyl)-L-Tyrosine PET for Treatment Management of Bevacizumab and Irinotecan in Patients with Recurrent High-Grade Glioma: A Cost-Effectiveness Analysis
}

\author{
Alexander Heinzel ${ }^{1,2}$, Dirk Müller ${ }^{3}$, Karl-Josef Langen ${ }^{2}$, Marcus Blaum $^{4}$, Frederik Anton Verburg ${ }^{1}$, Felix M. Mottaghy ${ }^{1}$, \\ and Norbert Galldiks 2,5 \\ ${ }^{1}$ Department of Nuclear Medicine, University of Aachen, Aachen, Germany; ${ }^{2}$ Institute for Neuroscience and Medicine, Research \\ Center Juelich, Juelich, Germany; ${ }^{3}$ Institute for Health Economics and Clinical Epidemiology, University of Cologne, Cologne, \\ Germany; ${ }^{4}$ Department of Neuroradiology, University of Aachen, Aachen, Germany; and ${ }^{5}$ Department of Neurology, University \\ of Cologne, Cologne, Germany
}

To date, the use of structural MR imaging (including contrast-enhanced and T2-weighted or fluid-attenuated inversion recovery-weighted images) is the standard method to diagnose tumor progression and to assess antiangiogenic treatment effects. However, several studies have suggested that $\mathrm{O}-\left(2-{ }^{18} \mathrm{~F}-\right.$ fluoroethyl)-L-tyrosine $\left({ }^{18} \mathrm{~F}-\mathrm{FET}\right)$ PET adds valuable clinical information to the information derived from structural MR imaging alone. We evaluated the effectiveness and cost-effectiveness of the addition of ${ }^{18} \mathrm{~F}$-FET PET to structural MR imaging for the management of treatment with bevacizumab and irinotecan $(\mathrm{BEV} / \mathrm{IR})$ in patients with recurrent high-grade glioma compared with MR imaging alone from the perspective of the German Statutory Health Insurance. Methods: To evaluate the incremental cost-effectiveness of the additional use of ${ }^{18} \mathrm{~F}-\mathrm{FET}$ PET, a decision tree model was used. Effectiveness of ${ }^{18} \mathrm{~F}$-FET PET was defined as correct identification of both tumor progression before $\mathrm{BEV} / \mathrm{IR}$ treatment initiation and BEV/IR treatment response and was evaluated for the combination of ${ }^{18} \mathrm{~F}$-FET PET and MR imaging compared with MR imaging alone. Costs were estimated for a baseline scenario and for a more expensive scenario. The robustness of the results was tested using deterministic and probabilistic sensitivity analyses. Results: The use of ${ }^{18}$ F-FET PET resulted in a number needed to diagnose of 2.4, that is, 3 additional patients have to be diagnosed to avoid 1 wrong diagnosis. The incremental costeffectiveness ratio of ${ }^{18} \mathrm{~F}$-FET PET/MR imaging compared with MR imaging alone was $€ 5,725(€ 1 \approx \$ 1.30)$ for the baseline scenario and $€ 8,145$ for the more expensive scenario per additional correct diagnosis. The probabilistic sensitivity analysis confirmed the robustness of the results. Conclusion: The model suggests that the additional use of ${ }^{18} \mathrm{~F}-\mathrm{FET}$ PET in the management of patients with recurrent high-grade glioma treated with BEV/IR may be cost-effective. Integration of ${ }^{18}$ F-FET PET has the potential to avoid overtreatment and corresponding costs, as well as unnecessary side effects to the patient.

Received Jan. 16, 2013; revision accepted Mar. 27, 2013

For correspondence or reprints contact: Alexander Heinzel, Department of Nuclear Medicine, University of Aachen, Pauwelsstrasse 30, 52074 Aachen, Germany.

E-mail: aheinzel@ukaachen.de

Published online Jun. 19, 2013.

COPYRIGHT (C 2013 by the Society of Nuclear Medicine and Molecular Imaging, Inc.
Key Words: ${ }^{18} \mathrm{~F}-\mathrm{FET}$ PET; cost-effectiveness analysis; recurrent glioma; decision tree model

J Nucl Med 2013; 54:1217-1222

DOI: 10.2967/jnumed.113.120089

$\mathbf{M}$ alignant gliomas are highly aggressive primary brain tumors, with an incidence of 7-8 per 100,000 people per year. Despite multimodal primary treatment including fluorescence-guided tumor resection and radiation therapy with concomitant temozolomide chemotherapy followed by adjuvant temozolomide chemotherapy, patients with glioblastoma, in particular, have an unfavorable prognosis (1). After tumor recurrence, the median survival ranges between 3 and 9 mo $(2,3)$.

Bevacizumab (Avastin; Genentech/Roche) is a humanized monoclonal antibody inhibiting the biologic activity of vascular endothelial growth factor. It is increasingly used as a single antiangiogenic agent or in combination with chemotherapy (e.g., topoisomerase 1 inhibitor irinotecan) in patients with recurrent high-grade glioma (rHGG) (4). Treatment with bevacizumab and irinotecan (BEV/IR) represents a promising option in patients with rHGG $(3,5)$. Furthermore, within a phase III multicenter study it has been used for patients with newly diagnosed glioblastoma (AVAglio trial). Bevacizumab given in combination with radiation and temozolomide was found to improve progression-free survival substantially (median improvement, $4.4 \mathrm{mo}$ ) (6).

To date, structural MR imaging is the most important diagnostic tool for assessing brain tumors because of its excellent soft-tissue contrast and multiplanar reconstruction capabilities $(7,8)$. However, structural MR imaging has important limitations in the assessment of treatment response or the identification of tumor recurrence. In addition, the extent of contrast enhancement on MR imaging (9) is used as an indicator of therapeutic response, although the reliability in distinguishing tumor tissue from unspecific treatment effects is limited. For example, after radiation therapy, reactive transient blood-brain barrier alterations with consecutive contrast enhancement can mimic tumor progression. This phenomenon, so-called pseudoprogression, is seen in 


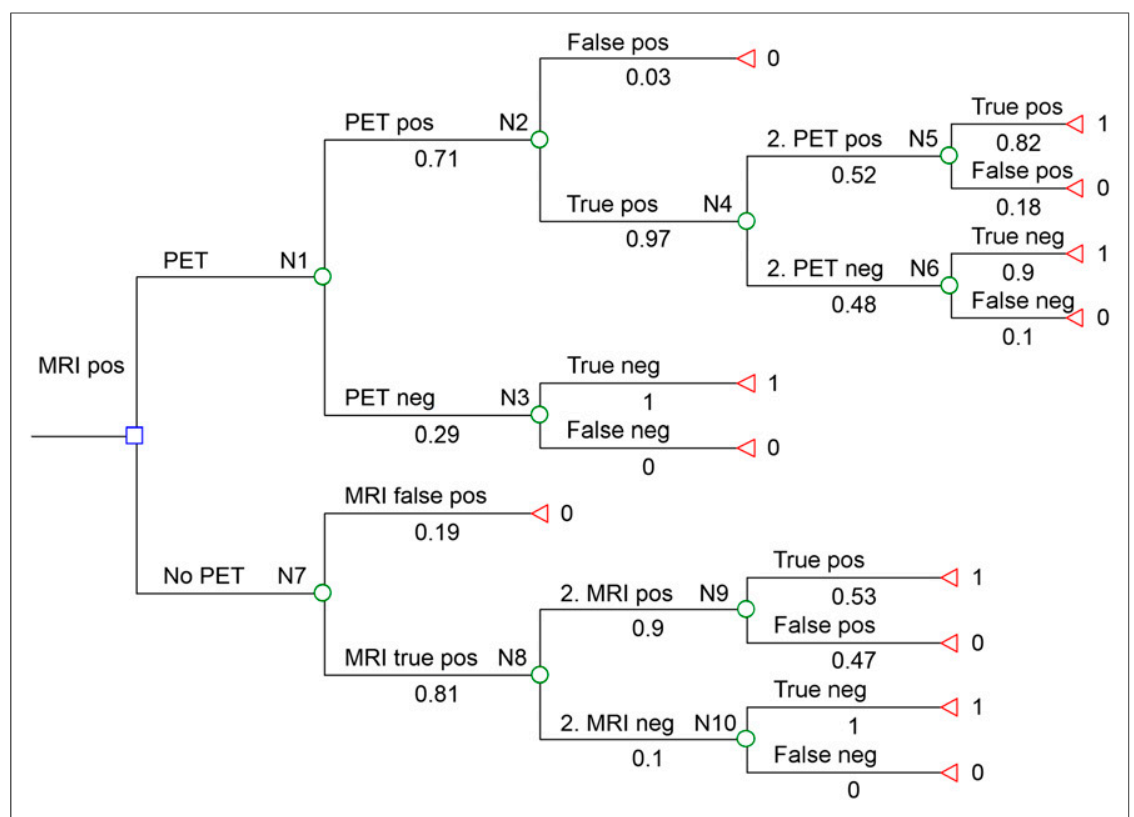

FIGURE 1. Decision tree model for assessment of effectiveness of additional ${ }^{18} \mathrm{~F}-\mathrm{FET}$ PET for management of treatment with BEV/IR in patients with rHGG. Model includes 2 alternative strategies using MR imaging alone or in addition to ${ }^{18} \mathrm{~F}-\mathrm{FET}$ PET. Probability of correct diagnostic assessment is defined as outcome. $\bigcirc=$ chance node; $\triangleleft=$ termination node; 2 . PET and 2. MRI = follow-up PET and MR imaging, respectively.

about $20 \%$ of cases and may result in unnecessary overtreatment (10)

Relevant to antiangiogenic drugs, the recently defined RANO (Response Assessment in Neuro-Oncology) criteria (11) recommend fluid-attenuated inversion recovery or $\mathrm{T} 2$ hyperintensity as a surrogate for nonenhancing tumor to support the determination of progression, and RANO thereby includes nonenhancing disease as a criterion for determining tumor response. However, nonenhancing tumor can be difficult to differentiate from other causes of fluidattenuated inversion recovery/T2 hyperintensity (e.g., radiationinduced gliosis, peritumoral edema, ischemia, or inflammation) (12).

Several $O-\left(2-{ }^{18} \mathrm{~F}\right.$-fluoroethyl $)$-L-tyrosine $\left({ }^{18} \mathrm{~F}-\mathrm{FET}\right)$ PET studies have demonstrated that ${ }^{18} \mathrm{~F}$-FET PET may offer additional valuable information in the management of patients with malignant gliomas (12-16). In comparison to structural MR imaging, it allows a more accurate estimation of size and extension of the metabolically active tumor $(13,14)$. For example, Rachinger et al. (15) directly compared the diagnostic performance of MR imaging and ${ }^{18} \mathrm{~F}$-FET PET in patients with predominantly rHGG. It could be observed that MR imaging is often insufficient to distinguish between benign posttherapeutic effects and tumor recurrence. Thus, in these patients ${ }^{18} \mathrm{~F}$-FET PET might improve the diagnosis of tumor recurrence. Additionally, further studies confirmed the utility of ${ }^{18} \mathrm{~F}$-FET PET in the diagnosis of $\mathrm{rHGG}(16,17)$.

Moreover, several studies demonstrated the applicability of amino acid PET tracers in the assessment of treatment response (18-20). Recently, 2 studies evaluated the use of ${ }^{18} \mathrm{~F}$-FET for monitoring treatment response to $\mathrm{BEV} / \mathrm{IR}(21,22)$. Both studies demonstrated that ${ }^{18}$ F-FET PET may allow a more reliable assessment of treatment response compared with MR imaging based on RANO criteria.

Based on these studies, we developed a decision tree model complemented by deterministic and probabilistic sensitivity analyses to evaluate the effectiveness and cost-effectiveness of using ${ }^{18} \mathrm{~F}$-FET
PET in addition to structural MR imaging for the management of treatment with BEV/ IR from the perspective of the Statutory Health Insurance in Germany. To our knowledge, no other studies have yet evaluated the cost-effectiveness of ${ }^{18} \mathrm{~F}$-FET PET in addition to structural MR imaging.

\section{MATERIALS AND METHODS}

\section{Decision Tree Model}

We built a decision tree model to analyze the effectiveness of adding ${ }^{18} \mathrm{~F}$-FET PET to structural MR imaging compared with using MR imaging alone for the management of treatment with BEV/IR in patients with $\mathrm{rHGG}$ (Fig. 1). The decision tree model was constructed and the sensitivity analyses performed using TreeAge Pro 2009 software (TreeAge Software, Inc.). The structure of the model is based on other models evaluating the cost-effectiveness of amino acid PET (23) and represents the common course of treatment and disease development according to 3 experts in the field.

The decision tree model (Fig. 1) includes the 2 alternatives: the use of additional ${ }^{18} \mathrm{~F}$ FET PET and the use of MR imaging alone. The model starts with the identification of tumor recurrence based on MR imaging. The decision tree model contains 10 chance nodes (N1-N10). The first chance node (N1) reflects a PET-positive and a PET-negative result, that is, the likelihood of tumor recurrence based on the additional information derived from the ${ }^{18} \mathrm{~F}-\mathrm{FET}$ PET scan. The chance nodes following the PET-positive/PET-negative branches (N2, N3) include the probability of a true- or false-positive and a true- or false-negative PET scan. In the case of a true-positive PET scan, tumor recurrence was assumed and treatment with BEV/IR was initiated. Follow-up PET was used to assess BEV/IR treatment response. The following branches represent the likelihoods of positive or negative PET scans (N4) and the likelihoods of true- or false-positive and true- or falsenegative PET scans (N5, N6). For follow-up ${ }^{18}$ F-FET PET, a positive result was defined as a positive treatment response.

In the approach using MR imaging alone to monitor BEV/IR treatment response, only positive MR imaging results were considered. The first branch (N7) represents the probability of a true- or false-positive MR imaging result. The following parts of the decision tree represent the assessment of treatment response using MR imaging. The followup MR imaging may again be true- or false-positive for the assessment of treatment response (N8). The following parts of the decision tree demonstrate the likelihoods of true- or false-positive results (N9, N10).

We defined the probability of a correct diagnosis as the primary outcome of our model. This appears to be an appropriate surrogate because it strongly influences decisions on treatment planning and monitoring. A wrong initial diagnosis of tumor recurrence will lead to premature aggressive treatment, with the risk of unnecessary serious side effects and avoidable costs for unnecessary treatment. The same applies to a wrong assessment of treatment response, which will similarly cause unnecessary serious side effects and may in addition prevent an early change of treatment strategy, with possible negative effects on the patients' life expectancy or quality of life.

\section{Diagnosis of Tumor Recurrence}

On the basis of the results of a previous study comparing the diagnostic value of contrast-enhanced MR imaging and ${ }^{18} \mathrm{~F}$-FET PET in 
patients with recurrent gliomas (15), we calculated for this study the positive predictive value (PPV) of both MR imaging and ${ }^{18} \mathrm{~F}$-FET PET and the negative predictive value (NPV) of ${ }^{18}$ F-FET PET. In that study, the PPV of MR imaging and ${ }^{18} \mathrm{~F}$-FET PET was 0.81 and 0.97 , respectively, whereas the NPV of ${ }^{18}$ F-FET PET was 1 . On the basis of this study, we assumed a likelihood of a positive PET scan of 0.71 . These values were applied in our decision tree model at the chance nodes N1, N2, N3, and N7 (Fig. 1).

\section{Treatment Monitoring}

Recently, 2 studies evaluated the potential of ${ }^{18}$ F-FET PET in comparison to MR imaging for treatment monitoring of BEV/IR in patients with rHGG $(21,22)$. Both studies contributed important additional ${ }^{18} \mathrm{~F}$ FET PET-derived information for treatment monitoring over and above the information obtained by MR imaging response assessment based on RANO criteria. The studies applied a similar research design and represent so far the only studies on this subject. The data of both studies were added and the diagnostic performance for MR imaging and ${ }^{18}$ F-FET PET based on these data was calculated (Table 1).

MR imaging-based RANO criteria use the following categories: complete response, partial response, stable disease, and progressive disease. For our analysis, we defined complete response, partial response, and stable disease as a treatment response indicating a positive effect of BEV/IR treatment. Progressive disease was defined as no treatment effect.

As described previously $(21,22)$, a reduction of the metabolically active tumor volume greater than $45 \%$ on follow-up ${ }^{18} \mathrm{~F}$-FET PET was considered a treatment response. Using a progression-free survival of 6 mo to differentiate long-term from short-term survivors, this threshold of metabolic response has been shown to be the optimal relation of sensitivity and specificity $(21,22)$.

On the basis of these data, we calculated the prevalence of positive scans, PPV, and NPV for both MR imaging and ${ }^{18}$ F-FET PET. For MR imaging, the prevalence of positive scans was 0.9 (N9), the PPV was 0.53 (N9), and the NPV was 1 (N10) (Fig. 1). For ${ }^{18}$ F-FET PET, the prevalence of positive scans was 0.52 (N4), the PPV was 0.82 (N5), and the NPV was 0.9 (N6) (Fig. 1).

\section{Calculation of the Costs}

The costs for ${ }^{18} \mathrm{~F}$-FET PET are currently not covered by the German Statutory Health Insurance. Therefore, the costs for ${ }^{18}$ F-FET were calculated using the reimbursement scheme for medical procedures for privately ensured patients (http://www.e-bis.de/goae/defaultFrame.htm). We calculated only the incremental costs for ${ }^{18} \mathrm{~F}$-FET since MR imaging is performed for all patients. A more detailed account of the calculation was described previously (23). Briefly, to reflect different levels of disease severity and related complexity of patient care, we calculated a baseline scenario and a severity-adjusted scenario. The baseline scenario contains the lowest reimbursement and represents the standard case. The severity-adjusted scenario has a higher reimbursement that allows adjusting for various factors such as the difficulty of the procedure or the qualifications of the health personnel. We included the following costs for the baseline and severity-adjusted scenarios, respectively $(€ 1 \approx \$ 1.30$ ): detailed patient consultation, $€ 8.74$ and $€ 20.10$; report on diagnostic findings, $€ 7.58$ and $€ 17.43$; intravenous injection, €4.08 and €9.38; whole-body tumor scintigraphy, €131.15 and $€ 236.07$; PET with quantitative analysis, €417.15 and €786.89. These categories refer to the codes $3,75,253,5431$, and 5489 of the

TABLE 1

Input Data for Treatment Monitoring

\begin{tabular}{|c|c|c|c|c|c|c|}
\hline \multirow[b]{2}{*}{ Patient no. } & \multicolumn{2}{|c|}{ MR imaging* } & \multicolumn{2}{|c|}{$\mathrm{PET}^{\dagger}$} & \multicolumn{2}{|c|}{ Progression-free survival $^{\ddagger}$} \\
\hline & Response & Status & Response & Status & Months & Status \\
\hline 1 & PR & 1 & $\mathrm{R}$ & 1 & 5 & 0 \\
\hline 2 & PD & 0 & NR & 0 & 3 & 0 \\
\hline 3 & PR & 1 & NR & 0 & 5 & 0 \\
\hline 4 & PD & 0 & NR & 0 & 2 & 0 \\
\hline 5 & PR & 1 & $\mathrm{R}$ & 1 & 4 & 0 \\
\hline 6 & PR & 1 & $\mathrm{R}$ & 1 & 10 & 1 \\
\hline 7 & PR & 1 & NR & 0 & 7 & 1 \\
\hline 8 & PR & 1 & NR & 0 & 4 & 0 \\
\hline 9 & SD & 1 & $\mathrm{R}$ & 1 & No progress & 1 \\
\hline 10 & SD & 1 & $\mathrm{R}$ & 1 & No progress & 1 \\
\hline 11 & PR & 1 & NR & 0 & 3 & 0 \\
\hline 12 & PR & 1 & NR & 0 & 4 & 0 \\
\hline 13 & PR & 1 & $\mathrm{R}$ & 1 & 12 & 1 \\
\hline 14 & PR & 1 & $\mathrm{R}$ & 1 & No progress & 1 \\
\hline 15 & PR & 1 & $\mathrm{R}$ & 1 & 12 & 1 \\
\hline 16 & PR & 1 & $\mathrm{R}$ & 1 & 6 & 1 \\
\hline 17 & SD & 1 & NR & 0 & 3 & 0 \\
\hline 18 & SD & 1 & NR & 0 & 3 & 0 \\
\hline 19 & SD & 1 & NR & 0 & 4 & 0 \\
\hline 20 & CR & 1 & $\mathrm{R}$ & 1 & 6 & 1 \\
\hline 21 & SD & 1 & $\mathrm{R}$ & 1 & 9 & 1 \\
\hline
\end{tabular}

${ }^{*}$ According to RANO criteria, $\mathrm{PR}=$ partial response, $\mathrm{PD}=$ progressive disease, $\mathrm{SD}=$ stable disease, and $\mathrm{CR}=$ complete response. Status $1=$ treatment effect (CR, PR, or SD), and status $0=$ no treatment effect (PD).

${ }^{\dagger}$ According to PET criteria, $\mathrm{R}=$ responder (reduction of metabolically active tumor volume $>45 \%$ ) and $\mathrm{NR}=$ nonresponder (volume reduction $<45 \%$ ). Status $1=R$, and status $0=N R$.

${ }^{\ddagger}$ Status $1=$ progression-free survival $\geq 6$ mo (successful treatment), and status $0=$ progression-free survival $<6$ mo.

Patient data are from Hutterer et al., 2011, and Galldiks et al., $2012(21,22)$. 
TABLE 2

Parameters Used in 1-Way Sensitivity Analyses and in Monte Carlo Simulations

\begin{tabular}{|c|c|c|}
\hline Parameter & $95 \%$ confidence interval & Distribution \\
\hline Node 1 & $0.7-1.0$ & Triangular (minimum, 0.7; likeliest, 0.79; maximum, 1) \\
\hline Node 2 & $0.84-1.0$ & Triangular (minimum, 0.84; likeliest, 0.97; maximum, 1) \\
\hline Node 3 & $0.85-1.0$ & Triangular (minimum, 0.85; likeliest, 0.95; maximum, 1) \\
\hline Node 4 & $0.4-0.6$ & Triangular (minimum, 0.4; likeliest, 0.52; maximum, 0.6) \\
\hline Node 5 & $0.7-0.9$ & Triangular (minimum, 0.7; likeliest, 0.82; maximum, 0.9) \\
\hline Node 6 & $0.85-0.95$ & Triangular (minimum, 0.85; likeliest, 0.9; maximum, 0.95) \\
\hline Node 7 & $0.60-0.85$ & Triangular (minimum, 0.75; likeliest, 0.81; maximum, 0.85) \\
\hline Node 8 & $0.70-0.90$ & Triangular (minimum, 0.70; likeliest, 0.85; maximum, 0.9) \\
\hline Node 9 & $0.5-0.7$ & Triangular (minimum, 0.5; likeliest, 0.53; maximum, 0.7) \\
\hline Node 10 & $0.8-1.0$ & Triangular (minimum, 0.8; likeliest, 0.95; maximum, 1) \\
\hline
\end{tabular}

Nodes N1-N10 refer to decision tree model in Figure 1.

above-mentioned cost scale. Additionally, the costs for the radioactive tracer have to be considered. Some hospitals using ${ }^{18}$ F-FET PET have the capacity to produce the tracers on-site. Moreover, ${ }^{18} \mathrm{~F}$-FET PET is available via commercial enterprises. To represent the range of costs, we calculated the mean of the price of 2 German enterprises and 1 onsite facility. Adding a value-added tax of $19 \%$, the resulting cost for the tracer was €616 (23).

Because the analysis was performed from the perspective of the German Statutory Health Insurance, we did not include indirect costs since they are irrelevant from the perspective of the health insurance. The cost-effectiveness of ${ }^{18}$ F-FET PET was calculated using the incremental cost-effectiveness ratio (24).

\section{Sensitivity Analyses}

To test the robustness of the results, we calculated deterministic and probabilistic sensitivity analyses. Deterministic 1-way sensitivity analyses were conducted for all values of the decision tree model to calculate the impact of their uncertainty. For each variable, 4 intervals were chosen. Because of a lack of published data, the low and the high values of the intervals were determined by questioning experts from nuclear medicine, neurooncology, and neuroradiology. The experts were asked to define a plausible range that contains $95 \%$ of the values for each variable (Table 2).

Probabilistic sensitivity analyses were performed using second-order Monte Carlo simulations with 10,000 samples (25). For the attribution of probability distributions to the variables of the decision tree, triangular distributions were used (23,26-28). On the basis of the initial definition of the intervals by the experts, we fitted the respective distributions for all variables (Table 2). Afterward, the experts were questioned again to ensure plausibility of the distributions.

\section{RESULTS}

The decision tree revealed that the use of ${ }^{18} \mathrm{~F}$-FET PET results in an increased rate of a correct diagnosis when compared with MR imaging alone $(P=0.881$ and $P=0.467$, respectively). Therefore, the rate of correct diagnoses increases by $41.4 \%$ when ${ }^{18} \mathrm{~F}$ FET PET is added to MR imaging alone. This means that 3 patients have to be diagnosed with ${ }^{18} \mathrm{~F}-\mathrm{FET}$ PET to avoid 1 wrong diagnosis (number needed to diagnose, $1 / 0.414=2.4$ ). The baseline scenario for two ${ }^{18} \mathrm{~F}$-FET PET scans results in total costs of $€ 2,370$ (morbidity-adjusted scenario, €3,372). The resulting incremental cost-effectiveness ratio is $€ 2,370 / 0.414=€ 5,725$ for the baseline scenario and $€ 3,372 / 0.414=€ 8,145$ for the severity-adjusted reimbursement rate scenario.

The results of the 1-way sensitivity analyses are shown in Table 3. The variation of N9 results in the lowest incremental effectiveness for ${ }^{18} \mathrm{~F}$-FET PET, at $28.9 \%$. This would lead to a number needed to diagnose of 3.5 , that is, 4 patients have to be diagnosed to avoid 1 wrong diagnosis. The highest incremental effectiveness was $53.4 \%$ (number needed to diagnose: 1.9 , that is, 2) resulting from variation of N7.

The statistics of the Monte Carlo simulations are presented in Table 4 and Figure 2. The incremental effectiveness of ${ }^{18} \mathrm{~F}-\mathrm{FET}$ PET compared with MR imaging resulted in a mean of $33.9 \%$. Within the interval of $95 \%$, the values range from $30.8 \%$ to $36.1 \%$, that is, the number needed to diagnose is between 2.77 and 3.25. The resulting incremental cost-effectiveness ratio is between $€ 6,565$ and $€ 7,695$ for the baseline scenario and between $€ 9,341$ and $€ 10,948$ for the severity-adjusted reimbursement rate.

TABLE 3

One-Way Sensitivity Analyses Based on Decision Tree Model

\begin{tabular}{llllll}
\hline \multicolumn{1}{c}{ Node } & \multicolumn{5}{c}{ Intervals } \\
\hline 1 & 0.700 & 0.775 & 0.850 & 0.925 & 1.000 \\
Incr eff & 0.415 & 0.403 & 0.390 & 0.378 & 0.365 \\
\hline Incr eff & 0.840 & 0.880 & 0.920 & 0.960 & 1.000 \\
3 & 0.852 & 0.357 & 0.381 & 0.406 & 0.431 \\
Incr eff & 0.370 & 0.888 & 0.925 & 0.963 & 1.000 \\
4 & 0.400 & 0.450 & 0.391 & 0.402 & 0.412 \\
Incr eff & 0.419 & 0.416 & 0.413 & 0.410 & 0.408 \\
5 & 0.700 & 0.750 & 0.800 & 0.850 & 0.900 \\
Incr eff & 0.369 & 0.387 & 0.405 & 0.423 & 0.441 \\
\hline 6 & 0.850 & 0.875 & 0.900 & 0.925 & 0.950 \\
Incr eff & 0.395 & 0.404 & 0.412 & 0.421 & 0.429 \\
7 & 0.600 & 0.663 & 0.725 & 0.788 & 0.850 \\
Incr eff & 0.534 & 0.498 & 0.462 & 0.426 & 0.390 \\
8 & 0.700 & 0.750 & 0.800 & 0.850 & 0.900 \\
Incr eff & 0.336 & 0.356 & 0.375 & 0.394 & 0.413 \\
9 & 0.500 & 0.550 & 0.600 & 0.650 & 0.700 \\
Incr eff & 0.434 & 0.398 & 0.362 & 0.325 & 0.289 \\
\hline 10 & 0.800 & 0.850 & 0.900 & 0.950 & 1.000 \\
Incr eff & 0.429 & 0.425 & 0.421 & 0.417 & 0.413 \\
\hline
\end{tabular}

Incr eff $=$ incremental effectiveness of additional ${ }^{18}$ F-FET PET compared with MR imaging alone.

Nodes N1-N10 refer to decision tree model in Figure 1. For each sensitivity analysis, 4 intervals were chosen. Low and high values are derived from probabilistic parameters in Table 1. 
TABLE 4

Monte Carlo Simulations on Effectiveness of Additional ${ }^{18}$ F-FET PET Compared with MR Imaging Alone for Treatment Management with BEV/IR

\begin{tabular}{lccc}
\hline \multicolumn{1}{c}{ Index } & ${ }^{18}$ F-FET PET & MR imaging alone & Incr eff \\
\hline Mean & 0.821 & 0.482 & 0.339 \\
SD & 0.032 & 0.046 & \\
Minimum & 0.673 & 0.339 & 0.334 \\
$2.5 \%$ & 0.754 & 0.393 & 0.361 \\
$10 \%$ & 0.778 & 0.421 & 0.357 \\
Median & 0.823 & 0.482 & 0.341 \\
$90 \%$ & 0.862 & 0.542 & 0.320 \\
$97.5 \%$ & 0.879 & 0.571 & 0.308 \\
Maximum & 0.919 & 0.642 & 0.277 \\
\hline
\end{tabular}

Data are probability of obtaining correct diagnosis using additional ${ }^{18} \mathrm{~F}$-FET PET or using MR imaging alone, applying 10,000 samples in Monte Carlo simulation. Incr eff $=$ incremental effectiveness of ${ }^{18} \mathrm{~F}$-FET PET compared with MR imaging alone.

\section{DISCUSSION}

This study evaluated the effectiveness and cost-effectiveness of additional ${ }^{18}$ F-FET PET for the management of treatment with BEV/IR. Compared with MR imaging alone, ${ }^{18} \mathrm{~F}$-FET PET increases the rate of correct diagnoses by $41.4 \%$ (resulting in a number needed to diagnose of 2.4), with an incremental cost-effectiveness ratio of $€ 5,725$ (baseline scenario) and $€ 8,145$ (severity-adjusted reimbursement rate scenario). The probabilistic sensitivity analysis confirmed the robustness of the results. The results of this study are in accordance with numerous publications that demonstrated the effectiveness of ${ }^{18}$ F-FET PET and other amino acid PET tracers for the management of patients with gliomas $(13,29,30)$.

Moreover, our results are also in line with other cost-effectiveness analyses evaluating the use of amino acid PET in patients with newly diagnosed gliomas $(23,27)$. The results of these studies suggested that amino acid PET might be cost-effective for planning the surgical resection of rHGG and for selecting the biopsy site for newly diagnosed brain tumors. ${ }^{18}$ F-FET PET cannot replace MR imaging but may provide cost-effective complementary information with important implications for the patients' management.

So far, no studies have evaluated direct patient-related benefits such as overall survival or an increase in quality of life as a result of the use of ${ }^{18} \mathrm{~F}$-FET. Because of this lack of evidence of direct patient-related benefits, we used the probability of a correct assessment of treatment response as the primary outcome of our model. This appears to be an appropriate surrogate since an incorrect diagnosis of the assessment of BEV/IR treatment response may lead to aggressive overtreatment with the risk of serious side effects and avoidable costs resulting from unnecessary treatment.

We assumed an increase in costs for using ${ }^{18} \mathrm{~F}$-FET PET of $€ 2,370$ for the baseline scenario and $€ 3,372$ for the severity-adjusted reimbursement rate scenario. This increase in costs appears acceptable considering the possibility of false-positive treatment assessment by MR imaging. Likewise, for the treatment with BEV/IR one may estimate about $€ 8,000$ (body weight, $80 \mathrm{~kg}$ ) per $4 \mathrm{wk}$ of treatment. Our analysis indicated that for the use of ${ }^{18} \mathrm{~F}-\mathrm{FET}$ PET the number needed to diagnose to obtain 1 additional correct diagnosis was 3 . Given the high treatment costs, a careful evaluation of the indication to treat may result in considerable savings by avoiding unnecessary treatment. Our analysis showed that the use of ${ }^{18}$ F-FET PET may contribute to this evaluation by carefully assessing the indication to start the treatment as well as by assessing the treatment success.

An important limitation of our study has to be noted. The clinical data applied in the decision tree model could be derived only from longitudinal within-group comparisons. The analysis on the use ${ }^{18}$ F-FET PET for diagnosis of tumor recurrence in patients with gliomas is based on the study of Rachinger et al. (15). That study is so far the biggest study that used ${ }^{18} \mathrm{~F}$-FET in patients with recurrent gliomas and included a direct comparison between the performance of ${ }^{18} \mathrm{~F}$-FET and MR imaging. However, the retrospective character of that study may lead to biased results.

The subsequent analysis of treatment response is based on 2 studies that represent so far the only studies on this subject. Both studies had small sample sizes, and none used a randomized controlled design. Therefore, additional studies are needed to confirm these results. As indicated by the 1-way sensitivity analyses, the probability of a true-positive or true-negative MR imaging result with regard to treatment monitoring has the strongest impact on the outcome. The decision tree model resulted in a number needed to diagnose of between 2 and 3, leading to an incremental cost-effectiveness ratio range between $€ 5,461$ and $€ 8,200$ for the baseline scenario (range of severity-adjusted reimbursement rate, $€ 7,770-€ 11,667)$. Thus, of great interest would be further studies that compare MR imagingbased and ${ }^{18}$ F-FET PET-based management of BEV/IR treatment in patients with rHGG, ideally performed in a randomized controlled trial. Moreover, studies addressing the comparison of MR imaging-based and ${ }^{18}$ F-FET PET-based diagnosis of tumor recurrence will additionally contribute to the empiric basis of the model.

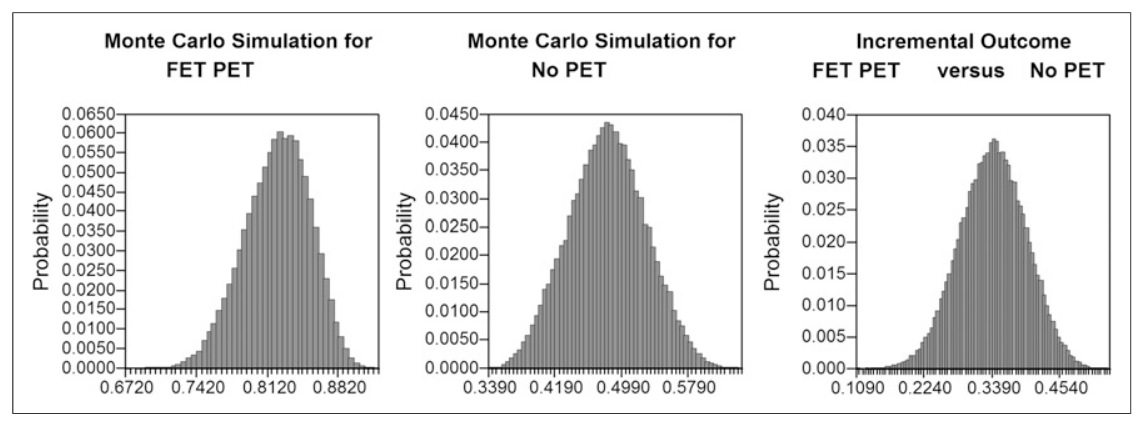

FIGURE 2. Distribution of results from Monte Carlo simulation with regard to effectiveness of additional ${ }^{18} \mathrm{~F}$-FET PET (left), effectiveness of MR imaging alone (middle), and distribution of incremental effectiveness (right).

\section{CONCLUSION}

The model delivers evidence that additional ${ }^{18} \mathrm{~F}$-FET PET may be cost-effective in the treatment management of BEV/IR in patients with rHGG. Additional studies are needed to confirm the results.

\section{DISCLOSURE}

The costs of publication of this article were defrayed in part by the payment of page charges. Therefore, and solely to indicate this fact, this article is hereby marked "advertisement" in accordance with 18 USC 
section 1734. No potential conflict of interest relevant to this article was reported.

\section{REFERENCES}

1. Stupp R, Mason WP, van den Bent MJ, et al. Radiotherapy plus concomitant and adjuvant temozolomide for glioblastoma. $N$ Engl J Med. 2005;352:987-996.

2. Wong ET, Hess KR, Gleason MJ, et al. Outcomes and prognostic factors in recurrent glioma patients enrolled onto phase II clinical trials. J Clin Oncol. 1999; $17: 2572-2578$

3. Xu T, Chen J, Lu Y, Wolff JE. Effects of bevacizumab plus irinotecan on response and survival in patients with recurrent malignant glioma: a systematic review and survival-gain analysis. BMC Cancer. 2010;10:252.

4. Friedman HS, Prados MD, Wen PY, et al. Bevacizumab alone and in combination with irinotecan in recurrent glioblastoma. J Clin Oncol. 2009;27:4733-4740.

5. Vredenburgh JJ, Desjardins A, Herndon JE II, et al. Bevacizumab plus irinotecan in recurrent glioblastoma multiforme. J Clin Oncol. 2007;25:4722-4729.

6. Chinot O, Wick W, Mason W, et al. Phase III trial of bevacizumab added to standard radiotherapy and temozolomide for newly-diagnosed glioblastoma: mature progression-free survival and preliminary overall survival results in AVAglio [abstract]. Neuro-Oncol. 2012;14:vi101-vi105.

7. Chen W. Clinical applications of PET in brain tumors. J Nucl Med. 2007;48: 1468-1481.

8. DeAngelis LM. Brain tumors. N Engl J Med. 2001;344:114-123.

9. Macdonald DR, Cascino TL, Schold SC Jr, Cairncross JG. Response criteria for phase II studies of supratentorial malignant glioma. J Clin Oncol. 1990;8: 1277-1280

10. Wick W, Wick A, Weiler M, Weller M. Patterns of progression in malignant glioma following anti-VEGF therapy: perceptions and evidence. Curr Neurol Neurosci Rep. 2011;11:305-312.

11. Wen PY, Macdonald DR, Reardon DA, et al. Updated response assessment criteria for high-grade gliomas: response assessment in neuro-oncology working group. J Clin Oncol. 2010;28:1963-1972.

12. Pope WB, Young JR, Ellingson BM. Advances in MR imaging assessment of gliomas and response to anti-VEGF therapy. Curr Neurol Neurosci Rep. 2011; 11:336-344.

13. Langen KJ, Hamacher K, Weckesser M, et al. O-(2-[ $\left[{ }^{18} \mathrm{~F}\right]$ fluoroethyl)-L-tyrosine: uptake mechanisms and clinical applications. Nucl Med Biol. 2006;33:287-294.

14. Pauleit D, Floeth F, Hamacher K, et al. O- $\left(2-\left[{ }^{18} \mathrm{~F}\right]\right.$ fluoroethyl $)$-L-tyrosine PET combined with MR imaging improves the diagnostic assessment of cerebral gliomas. Brain. Mar 2005;128:678-687.

15. Rachinger W, Goetz C, Popperl G, et al. Positron emission tomography with $\mathrm{O}-\left(2-\left[{ }^{18} \mathrm{~F}\right]\right.$ fluoroethyl)-l-tyrosine versus magnetic resonance imaging in the diagnosis of recurrent gliomas. Neurosurgery. 2005;57:505-511.
16. Mehrkens JH, Popperl G, Rachinger W, et al. The positive predictive value of $\mathrm{O}-\left(2-\left[{ }^{18} \mathrm{~F}\right]\right.$ fluoroethyl)-L-tyrosine (FET) PET in the diagnosis of a glioma recurrence after multimodal treatment. J Neurooncol. 2008;88:27-35.

17. Popperl G, Kreth FW, Herms J, et al. Analysis of ${ }^{18}$ F-FET PET for grading of recurrent gliomas: is evaluation of uptake kinetics superior to standard methods? J Nucl Med. Mar 2006;47:393-403.

18. Galldiks N, Langen KJ, Holy R, et al. Assessment of treatment response in patients with glioblastoma using $\mathrm{O}-\left(2-{ }^{18} \mathrm{~F}\right.$-fluoroethyl)-L-tyrosine PET in comparison to MR imaging. J Nucl Med. Jul 2012;53:1048-1057.

19. Pöpperl G, Goldbrunner R, Gildehaus FJ, et al. O-(2-[ $\left[{ }^{18} \mathrm{~F}\right]$ fluoroethyl)-L-tyrosine PET for monitoring the effects of convection-enhanced delivery of paclitaxel in patients with recurrent glioblastoma. Eur J Nucl Med Mol Imaging. 2005;32: 1018-1025.

20. Pöpperl G, Gotz C, Rachinger W, et al. Serial O-(2-[ $\left.{ }^{18} \mathrm{~F}\right]$ fluoroethyl)-L-tyrosine PET for monitoring the effects of intracavitary radioimmunotherapy in patients with malignant glioma. Eur J Nucl Med Mol Imaging. 2006;33:792-800.

21. Galldiks N, Rapp M, Stoffels G, et al. Response assessment of bevacizumab in patients with recurrent malignant glioma using $\left[{ }^{18} \mathrm{~F}\right]$ fluoroethyl-L-tyrosine PET in comparison to MRI. Eur J Nucl Med Mol Imaging. 2013;40:22-33.

22. Hutterer M, Nowosielski M, Putzer D, et al. O-(2- ${ }^{18}$ F-fluoroethyl)-L-tyrosine PET predicts failure of antiangiogenic treatment in patients with recurrent highgrade glioma. J Nucl Med. 2011;52:856-864.

23. Heinzel A, Stock S, Langen KJ, Muller D. Cost-effectiveness analysis of FET PET-guided target selection for the diagnosis of gliomas. Eur J Nucl Med Mol Imaging. 2012;39:1089-1096.

24. Karlsson G, Johannesson M. The decision rules of cost-effectiveness analysis. Pharmacoeconomics. 1996;9:113-120.

25. Doubilet P, Begg CB, Weinstein MC, Braun P, McNeil BJ. Probabilistic sensitivity analysis using Monte Carlo simulation: a practical approach. Med Decis Making. Summer 1985;5:157-177.

26. Burgos JL, Gaebler JA, Strathdee SA, Lozada R, Staines H, Patterson TL. Costeffectiveness of an intervention to reduce HIV/STI incidence and promote condom use among female sex workers in the Mexico-US border region. PLoS ONE. 2010;5:e11413.

27. Heinzel A, Stock S, Langen KJ, Muller D. Cost-effectiveness analysis of amino acid PET-guided surgery for supratentorial high-grade gliomas. J Nucl Med. 2012;53:552-558.

28. Meltzer MI, Dennis DT, Orloski KA. The cost effectiveness of vaccinating against Lyme disease. Emerg Infect Dis. 1999;5:321-328.

29. Singhal T, Narayanan TK, Jain V, Mukherjee J, Mantil J. ${ }^{11} \mathrm{C}-\mathrm{L}-$-methionine positron emission tomography in the clinical management of cerebral gliomas. Mol Imaging Biol. 2008;10:1-18.

30. Langen KJ, Floeth FW, Stoffels G, Hamacher K, Coenen HH, Pauleit D. Improved diagnostics of cerebral gliomas using FET PET [in German]. Z Med Phys. 2007; 17:237-241. 